\title{
Comparative Study of the Free Radical Scavenging Activities of Original and Generic Edaravone Determined by Electron Spin Resonance
}

\author{
Jimbo $\mathrm{H}^{1 *}$, Ikeda $\mathbf{Y}^{1}$ and Chang-il Lee $\mathbf{M}^{2}$
}

${ }^{1}$ Department of Neurosurgery, Tokyo Medical University Hachioji Medical Center, Tokyo, Japan

${ }^{2}$ Division of Pharmacology and ESR laboratories, Department of Clinical Care Medicine, Kanagawa Dental College, Yokosuga, Japan

\begin{abstract}
Edaravone, a powerful free radical scavenger, is the drug available in the clinical practice for the treatment of cerebral infarction and amyotrophic lateral sclerosis. Recently, many generic Edaravone injections have been commercialized. Substitution of additives in generic injections has the potential effect of changing the antioxidant ability of the original Edaravone injection. We investigated the dissimilarity between original and generic Edaravone injections focusing on their free radical scavenging activities determined by electron spin resonance spectroscopy. There were no significant differences between the original and generic Edaravone regarding the antioxidant abilities toward the hydroxyl radical. However, the generics in which the additive L-cysteine was substituted with glycerin or citric acid showed significant reduction in their antioxidant activity toward superoxide $(p<0.01)$. Our in vitro findings suggest that the antioxidant ability of generic Edaravone against the hydroxyl radical is equivalent to that of the original Edaravone and that substitution of additives in the generic Edaravone might change its antioxidant activity toward the superoxide.
\end{abstract}

Keywords: Edaravone; Antioxidant activity; Brain ischemia; Neuroprotectant

\section{Introduction}

Oxygen free radical species may play detrimental roles in brain ischemia and edema [1-3], and onset and progression of amyotrophic lateral sclerosis (ALS) [4]. Edaravone (3-methyl-1 phenyl-2-pyrazolin5-one, MCI-186, Radicut; developed by Mitsubishi Tanabe pharma corporation, Osaka, Japan), a powerful free radical scavenger, is currently used for the treatment of cerebral infarction and ALS [58]. It was shown that Edaravone scavenged radicals such as hydroxyl radical $\left(\mathrm{HO}^{*}\right)$, peroxyl radicals $\left(\mathrm{LO} 2{ }^{*}\right)$, DPPH (1,1-diphenyl-2picrylhydrazyl) radicals, and nitric oxide ( $\left.\mathrm{NO}^{*}\right)$ directly [5-7,9]. It was demonstrated that the inhibition of lipid peroxidation by Edaravone was probably due to the scavenging of the chain carrying $\mathrm{LO} 2^{\circ}$ and that, in combination with vitamin $\mathrm{E}$ or $\mathrm{C}$, the antioxidant activity of Edaravone may be enhanced [7]. In clinical studies, Edaravone improved the core neurological deficits, the activities of daily life, and the functional outcome of stroke patients $[10,11]$.

Recently, over 20 generic Edaravone injections have been put on the market in Japan. The generic injections should keep the same active ingredient, however, this rule cannot be applied to the additives. The additive L-cysteine present in the original Edaravone injections is substituted with citric acid, alpha thioglycerin, or glycine in some generic injections. The additives were changed in 10 generic Edaravone injections among 21 generics that have been approved in Japan in 2011 (Table 1). L-cysteine, citric acid, alpha thioglycerin, and glycine possess free radical scavenging activities, but their antioxidant potency is different. Hence, substitution of additives has the potential of changing the antioxidant ability of the original Edaravone injections.

In the present study, we investigated the dissimilarity between the original and generic Edaravone injections by focusing on their free radical scavenging activities determined by electron spin resonance (ESR) spectroscopy.

\section{Materials and Methods}

\section{Reagents}

The original Edaravone injection and 6 generic Edaravone injections are analyzed in this study. All the generics have the same active ingredient; however, the additives are different. Two generics employ glycine (A) and citric acid (E) instead of L-cysteine, while the other 4 generics have the same additives (Table 1). Solvents and other reagents are of the highest grade commercially available.

\section{Assay for radical intensity}

Superoxide $\left(\mathrm{O}_{2}{ }^{\circ}\right)$ was generated by titanium dioxide $\left(\mathrm{TiO}_{2}\right)$ photocatalysis, as described previously [12]. $\mathrm{HO}^{-}$was generated by the Fenton's reaction $\left(\mathrm{H}_{2} \mathrm{O}_{2} / \mathrm{FeSO}_{4}\right)$, as described previously $[13,14]$. Alternatively, $\mathrm{HO}$ could be generated by irradiating $\mathrm{H}_{2} \mathrm{O}$ with ultraviolet light ( $365 \mathrm{~nm} \mathrm{UV;} 5 \mathrm{~s} ; 40 \mathrm{~mW}$; SUPERCURE-203S, Radical Research, Tokyo, Japan), as described previously [15,16]. All the solutions were prepared in $0.1 \mathrm{M}$ phosphate -buffered saline (PBS) at $\mathrm{pH}$ 7.2. ESR spin trapping was conducted using a reactive oxygen species (ROS)-generating system containing 5-(2, 2-dimethyl-1,3propoxycyclophosphoryl)-5-methyl-1-pyrroline-N-oxide (CYMPO; Radical Research, Tokyo, Japan). ESR measurements were performed with a JES-RE1X system (JEOL, Tokyo, Japan) connected to the WINRAD ESR Data Analyzer program (Radical Research , Tokyo, Japan), under the following instrument settings: microwave power, $8.00 \mathrm{~mW}$; magnetic field $335.6 \pm 7.5 \mathrm{mT}$; field modulation width, $0.079 \mathrm{mT}$; sweep time, $1 \mathrm{~min}$; and time constant, $0.03 \mathrm{~s}$. All the experiments were repeated for a minimum of 3 times. Distilled water was used as the control. For each experimental group, the antioxidant activity was calculated as $100 \%$ with a mean value of control.

\section{Statistical analysis}

Statistical comparisons were made using non-repeated measures

*Corresponding author: Hiroyuki Jimbo, Department of Neurosurgery, Tokyo Medical University Hachioji Medical Center, 1163 Tatemachi Hachioji, Tokyo 1930998, Japan, Tel: 81426655611; Fax: 81426651796; E-mail: hjimbo@tokyo-med.ac.jp

Received July 15, 2015; Accepted August 18, 2015; Published August 21, 2015

Citation: Jimbo H, Ikeda Y, Chang-il LM (2015) Comparative Study of the Free Radical Scavenging Activities of Original and Generic Edaravone Determined by Electron Spin Resonance. J Neurol Disord 3: 245. doi:10.4172/2329-6895.1000245

Copyright: @ $2015 \mathrm{Jimbo} \mathrm{H}$, et al. This is an open-access article distributed under the terms of the Creative Commons Attribution License, which permits unrestricted use, distribution, and reproduction in any medium, provided the original author and source are credited. 


\begin{tabular}{|c|c|c|c|c|c|c|c|c|c|c|c|}
\hline \multicolumn{12}{|c|}{ Additives } \\
\hline & $\begin{array}{l}\text { sodium } \\
\text { hydrogen } \\
\text { sulfate }\end{array}$ & L-cysteine & $\begin{array}{l}\text { sodium } \\
\text { chloride }\end{array}$ & $\begin{array}{l}\text { sodium } \\
\text { hydroxide }\end{array}$ & $\begin{array}{l}\text { phosphoric } \\
\text { acid }\end{array}$ & $\begin{array}{l}\mathrm{pH} \\
\text { regulating } \\
\text { agent }\end{array}$ & $\begin{array}{l}\text { sodium } \\
\text { bicarbonate }\end{array}$ & glycine & citric acid & $\begin{array}{c}\text { sodium } \\
\text { citrate } \\
\text { hydrate }\end{array}$ & $\begin{array}{c}\text { alpha } \\
\text { thioglycerin }\end{array}$ \\
\hline Original & 0 & 0 & 0 & 0 & 0 & & & & & & \\
\hline \multicolumn{12}{|l|}{ Generics } \\
\hline A & 0 & & 0 & o & 0 & & & 0 & & & \\
\hline B & 0 & 0 & 0 & 0 & 0 & & & & & & \\
\hline C & o & 0 & 0 & 0 & 0 & & & & & & \\
\hline D & 0 & o & 0 & 0 & o & & & & & & \\
\hline E & 0 & & & 0 & & 0 & & & 0 & 0 & \\
\hline $\mathrm{F}$ & 0 & 0 & 0 & 0 & 0 & & & & & & \\
\hline $\mathrm{H}$ & 0 & & 0 & o & o & & & & & & o \\
\hline 1 & 0 & & 0 & 0 & 0 & & & & 0 & 0 & \\
\hline $\mathrm{J}$ & 0 & 0 & 0 & 0 & 0 & & & & & & \\
\hline K & 0 & 0 & 0 & 0 & 0 & & & & & & \\
\hline L & 0 & & 0 & 0 & 0 & & & & 0 & 0 & \\
\hline M & 0 & o & 0 & 0 & 0 & & & & & & \\
\hline $\mathrm{N}$ & 0 & & 0 & 0 & 0 & & & & & & 0 \\
\hline 0 & 0 & & 0 & 0 & 0 & & 0 & & & & \\
\hline$P$ & 0 & 0 & 0 & 0 & 0 & & & & & & \\
\hline$Q$ & 0 & & 0 & 0 & 0 & & & & 0 & & \\
\hline$R$ & 0 & 0 & 0 & 0 & 0 & & & & & & \\
\hline $\mathrm{s}$ & 0 & 0 & 0 & 0 & & 0 & & & & & \\
\hline $\mathrm{T}$ & 0 & o & 0 & & & o & & & & & \\
\hline$U$ & 0 & & 0 & & & 0 & & & & & 0 \\
\hline V & 0 & & o & 0 & & o & & & 0 & 0 & \\
\hline
\end{tabular}

Table 1: Changes of additives in generic Edaravone injections which have been approved in Japan at 2011.

analysis of variance (ANOVA). Data are expressed as mean \pm standard deviation (SD). The statistical significance was set at $p<0.05$.

\section{Results}

\section{Dissimilarity between the original and generic Edaravone on $\mathrm{O}_{2}$.}

We investigated the antioxidant effect of the original and generic Edaravone on $\mathrm{O}_{2}{ }^{-}$generated by $\mathrm{TiO}_{2}$ photocatalysis using the ESR spin trapping technique with CYMPO. The significant reduction in the antioxidant activity toward $\mathrm{O}_{2}{ }^{\circ}$ in the case of 2 generic Edaravone injections compared with that of the original drug was measured ( $p$ $<0.01)$. There were no significant differences in the generics in which the additives were equivalent to those of the original injection (generic Edaravone B, C, D and F); however, the generics $\mathrm{A}$ and $\mathrm{E}$ in which the additive L-cysteine was substituted with glycerin or citric acid respectively showed reduction in their antioxidant activity toward $\mathrm{O}_{2}{ }^{\circ}$ (Figure 1).

\section{Dissimilarity between the original and generic Edaravone on HO}

We investigated the antioxidant activity of the original and generic Edaravone on $\mathrm{HO}^{*}$ generated by Fenton's reaction or ultraviolet irradiation of $\mathrm{H}_{2} \mathrm{O}_{2}$, using ESR spin trapping with CYMPO. There were no significant differences between the original and generic Edaravone injections (Figures 2 and 3).

\section{Discussion}

The relationship between ischemic brain injury and lipid peroxidation disorder by free radicals was first described by Flamm et al. in 1978 [2]. Increasing evidence suggests that oxygen free radical species may play detrimental roles in ischemic diseases [1,3,17-19] and ALS [4] and selective free radical scavengers as brain protective agents are considered to be potential drugs against brain ischemia [20] and ALS

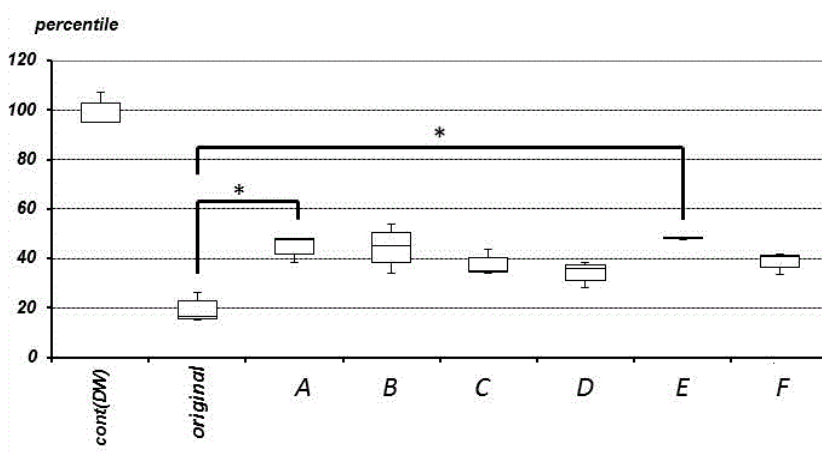

Figure 1: Antioxidant activity of the original and generic Edaravone injections toward superoxide $\left(\mathrm{O}_{2}{ }^{-}\right)$generated by the $\mathrm{TiO}_{2}$ photocatalysis reaction calculated using the relative ESR signal intensities. The data are expressed as mean \pm standard deviation (SD, $n=9$ ) calculated as $100 \%$ with the mean value of distilled water as the control. The additives were substituted from $\mathrm{L}$-cysteine to glycine in $\mathrm{A}$, and to citric acid in $\mathrm{E}$ (Table 1). The significant differences compared to the original injection were recognized in $A$ and E. A-F: Generic Edaravone injections which have been made in each pharmaceutical company, DW: distilled water, cont: control, *: Significantly different $(p<0.01)$ compared to the original injection

[8]. Although the world's first clinical use of Edaravone, which has been developed as a neuroprotectant, was approved in Japan in 2001 [11], it remains under clinical investigation in various other countries [21] and its use has not been approved yet in Western countries. However, Edaravone is recommended by the American Heart Association in the guidelines for the early management of adults with acute ischemic stroke [22]. The administration of Edaravone during Alteprase infusion is likely to enhance the recanalization in patients with acute ischemic stroke [23]. The generic Edaravone injections have been approved in 


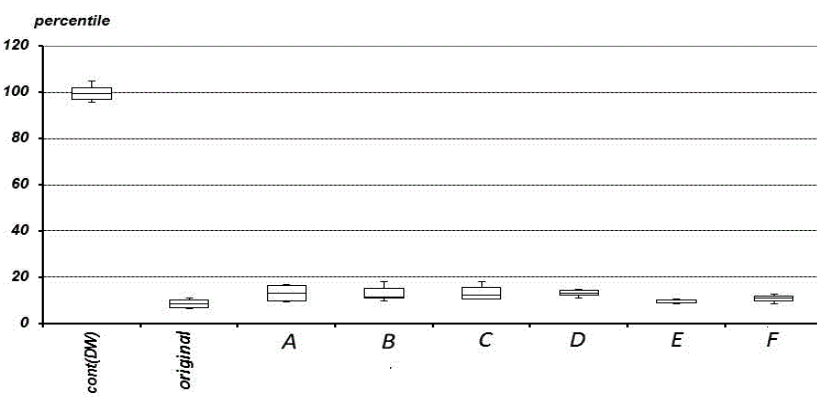

Figure 2: Antioxidant activity of the original and generic Edaravone injections towards the hydroxyl radical $\left(\mathrm{HO}^{*}\right)$ generated by the Fenton reaction calculated using the ESR signal intensities. The data are expressed as mean \pm standard deviation $(\mathrm{SD}, \mathrm{n}=9$ ) calculated as $100 \%$ with the mean value of distilled water as the control. There were no significant differences between the original and generic injections. A-F: Generic Edaravone injections which have been made in each pharmaceutical company, and the additives were substituted from L-cysteine to glycine in A and to citric acid in E, DW: distilled water, cont: control

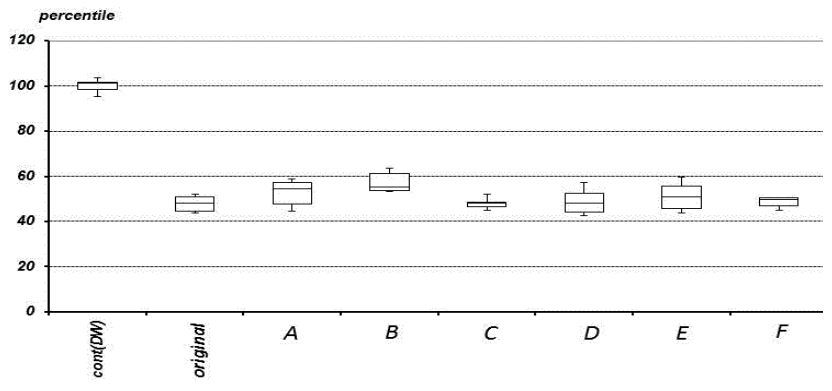

Figure 3: Antioxidant activity of the original and generic Edaravone injections toward the hydroxyl radical $\left(\mathrm{HO}^{*}\right)$ generated by the ultraviolet irradiation of $\mathrm{H}_{2} \mathrm{O}_{2}$, calculated using the ESR signal intensities. The data are expressed as mean \pm standard deviation ( $S D, n=9$ ) calculated as $100 \%$ with the mean value of distilled water as the control. There were no significant differences between the original and generic injections. A-F: Generic Edaravone injections which have been made in each pharmaceutical company and the additives were substituted from L-cysteine to glycine in A and to citric acid in E, DW: distilled water, cont: control

Japan in 2011 and are now produced in several countries such as Korea, India, and China.

The basic chemical structure of Edaravone (3-Methyl-1-phenyl2-pyrazolin-5-one) was found to show promising activity as an antioxidative radical scavenger, was capable of quenching $\mathrm{HO}^{\circ}$, which among other oxidative free radicals has the strongest oxidative ability, and could inhibit both $\mathrm{HO}^{\circ}$ - dependent and $\mathrm{HO}^{\circ}$ - independent lipid peroxidation. Furthermore, additional free radical scavenging and antioxidative actions of Edaravone were identified in $\mathrm{LOO}^{\circ}$ - induced peroxidation [7] and peroxynitrite ( $\left.\mathrm{ONOO}^{-}\right)$-induced tyrosine nitration [6,9]. However, this basic chemical structure of Edaravone does not show antioxidant activity for $\mathrm{O}_{2}^{-}$, which has very low oxidative ability [6]. In our study, the ability of the generic Edaravone as a free radical scavenger for $\mathrm{HO}$ 'was found to be equivalent to that of the original drug, proving the safety of the basic chemical structure of the generic Edaravone. Therefore, the generic Edaravone drugs have achieved the objective of the development of original Edaravone equally.

Whether Edaravone could scavenge $\mathrm{O}_{2}{ }^{\circ}$ or not is still controversial [24]. It is known that the thiol group (-SH) of L-cysteine reacts with the $\mathrm{DPPH}$ radical and thus scavenges free radicals $[25,26]$. Consistently, a significant reduction in the $\mathrm{O}_{2}{ }^{\circ}$ scavenging activity was found only in those generics in which the additive L-cysteine was substituted with glycerin or citric acid. Even though $\mathrm{O}_{2}{ }^{-}$has low oxidative ability, it is the key radical that initiates other free radical chain reactions. The fact that the substitution of additives affected the scavenging activity of Edaravone for $\mathrm{O}_{2}{ }^{\circ}$ showed that L-cysteine plays an important role in scavenging $\mathrm{O}_{2}{ }^{-}$in Edaravone injections. There is a report mentioned that $1 \mathrm{mM}$ Edaravone injection suppressed the formation of $\mathrm{O}_{2}{ }^{-}$in the xanthine oxidase-hypoxanthine system [24]. Therefore, the generic Edaravone injections in which additive L-cysteine has been substituted with another additive have the possibility to reduce its own ability as an antioxidative radical scavenger. However, our study is an in vitro analysis, while the additives injected into the veins are immediately diluted and spread by a large amount of blood; therefore, in vivo, the radical scavenging activity of L-cysteine might disappear. Further in vivo studies are necessary to draw the conclusion that additive changes in generic Edaravone involve the antioxidant ability in clinical use.

\section{Conclusion}

Our in vitro findings suggest that the antioxidant ability of generic Edaravone against the $\mathrm{HO}^{\circ}$ radical is equivalent to that of the original Edaravone and that substitution of additives in the generic Edaravone might change its antioxidant activity toward the $\mathrm{O}_{2} \cdot$.

\section{Acknowledgments}

We gratefully acknowledge Takeo Otsuka, Makiko Saida, Tomoko Komatsu Ayaka Yoshida, and Fumihiko Yoshino for their excellent technical assistance.

\section{References}

1. Demopoulos HB, Flamm ES, Pietronigro DD, Seligman ML (1980) The free radical pathology and the microcirculation in the major central nervous system disorders. Acta Physiol Scand Suppl 492: 91-119.

2. Flamm ES, Demopoulos HB, Seligman ML, Poser RG, Ransohoff J (1978) Free radicals in cerebral ischemia. Stroke 9: 445-447.

3. Siesjö BK (1981) Cell damage in the brain: a speculative synthesis. J Cereb Blood Flow Metab 1: 155-185.

4. Beckman JS, Carson M, Smith CD, Koppenol W (1993) ALS, SOD and peroxynitrite. Nature 364: 584.

5. Watanabe K, Yuki S, Saito K, Sato S, Sugimoto J, et al. (1997) Pharmacological studies of MCl-186, a new drug for acute stroke, Jpn Pharmacol Ther 25: 1691 1698.

6. Watanabe K, Watanabe K, Hayase T (1997) Radical scavenging mechanism of MCl-186, Jpn Pharmacol Ther 25: 1699-1707.

7. Yamamoto Y, Kuwahara T, Watanabe K, Watanabe K (1996) Antioxidant activity of 3-methyl-1-2-pyrazoline-5-0ne. Redox Report 2: 333-338.

8. Abe K, Itoyama Y, Sobue G, Tsuji S, Aoki M, et al. Confirmatory double-blind, parallel-group, placebo-controlled study of efficacy and safe of edaravone (MCl-186) in amyotrophic lateral sclerosis patients. Amyotrophic Lateral Sclerosis and Frontotemporal Degeneration 15: 610-617.

9. Satoh K, Ikeda Y, Shioda S, Tobe T, Yoshikawa T (2002) Edarabone scavenges nitric oxide. Redox Rep 7: 219-222.

10. Edaravone Acute Infarction Study Group (2003) Effect of a novel free radical scavenger, Edaravone (MCl-186), on acute brain infarction. Randomized, placebo-controlled, double-blind study at multicenters. Cerebrovasc Dis 15 222-229.

11. Houkin K, Nakayama N, Kamada K, Noujou T, Abe H, et al. (1998) Neuroprotective effect of the free radical scavenger $\mathrm{MCl}-186$ in patients with cerebral infarction: clinical evaluation using magnetic resonance imaging and spectroscopy. J Stroke Cerebrovasc 7: 315-322.

12. Lee MC, Yoshino F, Shoji H, Takahashi S, Todoki K, et al. (2005) Characterization by electron spin resonance spectroscopy of reactive oxygen species generated by titanium dioxide and hydrogen peroxide. J Dent Res 84: 178-182. 
Citation: Jimbo H, Ikeda Y, Chang-il LM (2015) Comparative Study of the Free Radical Scavenging Activities of Original and Generic Edaravone Determined by Electron Spin Resonance. J Neurol Disord 3: 245. doi:10.4172/2329-6895.1000245

Page 4 of 4

13. Hagiwara T, Lee $\mathrm{Cl}$, Okabe E (2000) Differential sensitivity to hydroxyl radicals of pre- and postjunctional neurovascular transmission in the isolated canine mesenteric vein. Neuropharmacology 39: 1662-1672.

14. Lee C, Okabe $\mathrm{E}$ (1995) Hydroxyl radical-mediated reduction of $\mathrm{Ca}(2+)$-ATPase activity of masseter muscle sarcoplasmic reticulum. Jpn J Pharmacol 67: 2128.

15. Ogasawara Y, Namai T, Yoshino F, Lee MC, Ishii K (2007) Sialic acid is an essential moiety of mucin as a hydroxyl radical scavenger. FEBS Lett 581 : 2473-2477.

16. Sakurai K, Sasabe H, Koga T, Konishi T (2004) Mechanism of hydroxyl radical scavenging by rebamipide: identification of mono-hydroxylated rebamipide as a major reaction product. Free Radic Res 38: 487-494.

17. Lewén A, Matz P, Chan PH (2000) Free radical pathways in CNS injury. J Neurotrauma 17: 871-890.

18. Morimoto T, Globus MY, Busto R, Martinez E, Ginsberg MD (1996) Simultaneous measurement of salicylate hydroxylation and glutamate release in the penumbral cortex following transient middle cerebral artery occlusion in rats. J Cereb Blood Flow Metab 16: 92-99.

19. Peters O, Back T, Lindauer U, Busch C, Megow D, et al. (1998) Increased formation of reactive oxygen species after permanent and reversible middle cerebral artery occlusion in the rat. J Cereb Blood Flow Metab 18: 196-205.

20. Tamura A, Asano T, Sano K, Tsumagari T, Nakajima A (1979) Protection from cerebral ischemia by a new imidazole derivative (Y-9179) and pentobarbital. A comparative study in chronic middle cerebral artery occlusion in cats. Stroke 10: $126-134$

21. Wang CX, Shuaib A (2007) Neuroprotective effects of free radical scavengers in stroke. Drugs Aging 24: 537-546.

22. Adams HP, del Zoppo G, Alberts MJ (2007) Guidelines for the early management of adults with ischemic stroke: A guideline from the American Heart Association/ American Stroke Association Stroke Council, Clinical Cardiology Council, Cardiovascular Radiology and Intervention Council, and the Atherosclerotic Peripheral Vascular Disease and Quality of Care Outcomes in Research Interdisciplinary Working Groups: The American Academy of Neurology affirms the value of this guideline as an educational tool for neurologists. Circulation 22: e478-e534.

23. Kimura K, Aoki J, Sakamoto Y, Kobayashi K, Sakai K, et al. (2012) Administration of edaravone, a free radical scavenger, during t-PA infusion can enhance early recanalization in acute stroke patients--a preliminary study. J Neurol Sci 313: 132-136.

24. Wu TW, Zeng LH, Wu J, Fung KP (2000) MCl-186: further histochemical and biochemical evidence of neuroprotection. Life Sci 67: 2387-2392.

25. Molyneux $P$ (2004) The use of the stable free radical diphenylpicrylhyd razy (DPPH) for estimating antioxidant activity. Songklanakarin J Sci Technol 26 211-219.

26. Yamaguchi T, Takamura H, Matoba T, Terao J (1998) HPLC method for evaluation of the free radical-scavenging activity of foods by using, 1-diphenyl2-picrylhydrazyl. Biosci Biotechnol Biochem 62: 1201-1204. 